\title{
Eukaryotic Initiation Factor-2, Gamma Subunit, Suppresses Proliferation and Regulates the Cell Cycle via the MAPK/ERK Signaling Pathway in Acute Myeloid Leukemia
}

\author{
Jielun Lu \\ Guangzhou Medical College First Affiliated Hospital \\ SHUYI CHEN \\ Guangzhou Medical College First Affiliated Hospital \\ HUO TAN \\ Guangzhou Medical College First Affiliated Hospital \\ ZHENQIAN HUANG \\ Guangzhou Medical College First Affiliated Hospital \\ BO LI \\ Guangzhou Medical College First Affiliated Hospital \\ LING LIU \\ Guangzhou Medical University Second Affiliated Hospital \\ YIMIN CHEN \\ Guangzhou Medical College First Affiliated Hospital

\section{XIAOZHEN ZENG} \\ Guangzhou Medical College First Affiliated Hospital \\ YAWEI ZOU \\ Guangzhou Medical College First Affiliated Hospital \\ LIHUAXU ( $\nabla$ xlhua@gzhmu.edu.cn) \\ Guangzhou Medical University Affiliated Cancer Hospital
}

\section{Research Article}

Keywords: EIF2S3, cellular proliferation, the cell cycle, prognosis, acute myeloid leukemia, MAPK/ERK signaling pathway

Posted Date: April 6th, 2021

DOI: https://doi.org/10.21203/rs.3.rs-371475/v1

License: (c) (i) This work is licensed under a Creative Commons Attribution 4.0 International License. Read Full License

Version of Record: A version of this preprint was published at Journal of Cancer Research and Clinical Oncology on July 7th, 2021. See the published version at https://doi.org/10.1007/s00432-021-03712-5. 


\section{Abstract}

Purpose The expression of eukaryotic translation initiation factor 2 subunit 3 (EIF2S3) in patients with non-small cell lung and colorectal cancer is lower than that in healthy individuals. However, the functions of EIF2S3 remain unclear, and its study in leukemia has not been reported. The article aims to explore the role of EIF2S3 in AML (acute myeloid leukemia) and its underlying mechanism.

Methods Reverse transcription-quantitative PCR was performed to evaluate the expression levels of EIF2S3, and its association with patient prognosis was determined. Inducible HEL-EIF2S3 and HL-60-EIF2S3 cell lines were established by retrovirus infection. Cellular proliferation and the cell cycle were analyzed using Cell Counting Kit 8 and flow cytometric analyses. Tumorigenic ability was evaluated using xenograft nude mouse model. Gene expression profiles were analyzed in HL-60-EIF2S3 cells by next-generation sequencing, and WB analysis was performed to detect the expression of related proteins.

Results The expression of EIF2S3 in patients with AML was lower than that experiencing $C R(P=0.02)$. Furthermore, EIF2S3 overexpression inhibited cellular proliferation, halted G0/1 to $S$ phase cell cycle progression and inhibited tumorigenicity $(P=0.015)$. 479 differentially expressed genes were identified between HL60-EIF2S3 DOX (-) and HL60-EIF2S3 DOX (+) cells via NGS and several of them involved in MAPK/ERK signaling pathway. The phosphorylation levels of ERK decreased when EIF2S3 was overexpressed(P凶0.050).

Conclusion EIF2S3 overexpression may result in a decrease in cellular proliferation, cell cycle arrest and tumorigenic inhibition via the MAPK/ERK signaling pathway in AML cells.

\section{Introduction}

Acute myeloid leukemia (AML) is a myeloid malignancy characterized by the aberrant proliferation of immature and abnormal primitive cells, and the disruption of hematopoiesis (1). As a common type of leukemia, the American Cancer Society estimated 19,520 new cases of AML in the United States, accounting for 1.2\% of all new cancer cases in 2019 (2). In China, leukemia was one of the top ten malignancies with a high mortality rate, with an increasing incidence rate from 2000 to 2011 (3). With the rapid development of cytogenetics and molecular biological techniques, constant progress has been made in the diagnosis and treatment of AML in recent years. In addition to the traditional treatments of chemotherapy and hematopoietic stem cell transplantation, various novel moleculartargeted agents have been identified, including histone deacetylase and receptor-type tyrosine-protein kinase FLT3 inhibitors (4). The Surveillance, Epidemiology, and End Results Program have demonstrated that the relative 5-year survival rate increased from 7.41 to $29.77 \%$ in patients with AML, though this is still a relatively low rate in general (2). Furthermore, relapse remained the primary reason for this poor prognosis (5). Therefore, the focus of AML therapy is to identify novel biomarkers for improving treatment options and patient prognosis.

Eukaryotic translation initiation factor 2 subunit 3 (EIF2S3/elF2 $\gamma$ ) is located in the Xp22.11 region of the X chromosome, and comprises 472 amino acids. EIF2S3 is a protein subunit that contains a GTP-binding pocket. As the core of the heterotrimer, EIF2S3 constitutes the eukaryotic translation initiation factor 2 (elF2) with the other two subunits, EIF2S1 and EIF2S2 (6-8). elF2 plays an important role in eukaryotic translation, binding GTP through elF2 $\gamma$ and initiator methionyl-tRNA (Met-tRNA ${ }_{i}$ Met ) to form a stable ternary complex, and identifying the translation initiation site $(7,9)$. Previous studies have reported that EIF2S3 mutations cause X-linked intellectual disabilities (XLID; also known as mental retardation, epileptic seizures, hypogenitalism, microcephaly and obesity syndrome, based on its associated characteristics), characterized by mental deficiency, epilepsy, hypogenitalism, microcephaly and obesity (10-13). In tumor-related studies, Chain et al (14) illustrated that the level of peripheral blood EIF2S3 mRNA expression was lower in patients with non-small cell lung cancer than in healthy subjects. Chang et al. (15) observed a similar outcome in colorectal cancer. However, the functions and mechanisms underlying these observations remain unclear, and there are currently no relevant reports surrounding EIF2S3 expression in AML.

To the best of our knowledge, the present study was the first to discover that EIF2S3 is significantly associated with clinicopathological parameters and a positive prognosis in patients with AML. EIF2S3 overexpression resulted in a decrease in cellular proliferation, cell cycle arrest and tumorigenic inhibition via the MAPK/ERK signaling pathway.

\section{Materials And Methods}

Patients and samples. A total of 61 bone marrow (BM) samples were acquired from patients with AML at the First Affiliated Hospital of Guangzhou Medical University (Guangzhou, China), who were diagnosed between October 2012 and March 2017. Diagnosis was established following clinical, morphological, cytochemical, flow cytometric and cytogenetic analysis, which conformed to the World Health Organization (WHO) diagnostic criteria $(16,17)$. A marrow or blood blast count $\geq 20 \%$ was considered as diagnostic of AML, except

Page 2/16 
for patients with $\mathrm{t}(15 ; 17), \mathrm{t}(8 ; 21)$, inv(16) or $\mathrm{t}(16 ; 16)$. Complete remission (CR) was defined as follows: i) A BM blast count <5\%; ii) absence of blasts with Auer rods; iii) absence of extramedullary disease; iv) absolute neutrophil counts $>1.0 \times 10^{9} / \mathrm{l}$; v) platelet counts $>100 \times 10^{9} /$; and vi) independence of red cell transfusions (18). According to the French-American-British (FAB) classification for the diagnosis and classification of AML (17), 41 samples were classified as primary AML, including 1 sample with M0, 3 samples with M1, 12 samples with M2, 6 samples with M3, 7 samples with M4, 7 samples with M5, 1 sample with $M 6$ and 4 unclassified samples. A further 20 samples were obtained from patients with CR. The follow-up time ended in July 2018. The study was approved by the ethics committee of the First Affiliated Hospital of Guangzhou Medical University, and each patient gave written informed consent for the use of their specimens for medical research, conforming to the tenets of the Declaration of Helsinki.

Cell culture. AML cell lines (HL-60 and HEL) and 293FT cells were purchased from the cell library of the Chinese Academy of Sciences, and separately cultured in RPMI 1640 medium (HL-60 and HEL cells) and DMEM (293FT cells) (both HyClone; Cytiva) with 10\% fetal bovine serum (FBS; Gibco; Thermo Fisher Scientific, Inc.). The cells were maintained in an incubator at $37^{\circ} \mathrm{C}$ with $5 \% \mathrm{CO}_{2}$ and $95 \%$ air.

Vector construction. Cells overexpressing EIF2S3 were generated using the Retro-X Tet-Off Advanced doxycycline (DOX) inducible expression system per the manufacturer's protocol (TaKara Biotechnology Co., Ltd.). The targeted gene (EIF2S3) was suppressed in the presence of $100 \mathrm{ng} / \mathrm{ml}$ DOX (Sigma-Aldrich; Merck KGaA). The full-length coding sequence of the EIF2S3 gene was determined using the NCBI (https://www.ncbi.nlm.nih.gov/gene/) and amplified using KOD DNA polymerase (Toyobo Life Science) with the following primers: EIF2S3 forward, 5'-ATAAGAATGCGGCCGCATGGCGGGCGGAGAA-3' and reverse, 5'- GGAATTCTCAGTCATCATCTACTGTTGGC-3'. The

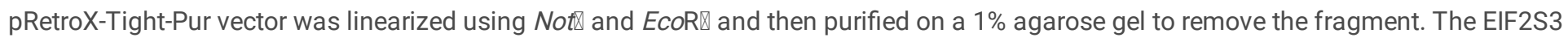
gene fragment was cloned into the linearized vector (designated pRetroX-Tight-EIF2S3) using the Ligation high kit (Toyobo Life Science).

Transformation. transfection and stable cell line generation. Competent DH5a cells (TransGen Biotech Co., Ltd.) were transformed with the recombinant pRetroX-Tight-EIF2S3 vector. The cells were cultured on amp-agarose plates overnight (16-24 h) at $37^{\circ} \mathrm{C}$. Colonies were then

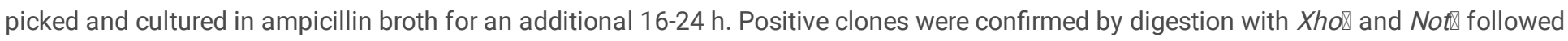
by sequencing. pRetroX-Tight-EIF2S3, pRetroX-Tight-Pur, pRetro-X Tet-Off Advanced and the packing plasmid pCL-Ampho were cotransfected into 293FT cells using TransIntro ${ }^{\text {TM }}$ EL Transfection Reagent (TransGen Biotech Co., Ltd) per the manufacturer's protocol. At 48 h post-transfection, the culture media were clarified using a $0.45-\mu \mathrm{m}$ filter and the viral supernatant was harvested; HL-60 and HEL cells were infected following the addition of $4 \mu \mathrm{g} / \mathrm{ml}$ polybrene. After $6 \mathrm{~h}$, the media were replaced, and the cells were incubated for a further 24 h. Stably transfected cells were selected with puromycin $(1 \mu \mathrm{g} / \mathrm{ml}$ for $72 \mathrm{~h})$. Cultured cells were induced with DOX (100 $\mathrm{ng} / \mathrm{ml})$ for $2 \mathrm{days}$ and protein expression were assessed by western blotting. Cells were induced for a further 4 days prior to use in experiments.

RNA extraction and reverse transcription-quantitative PCR (RT-qPCR). Mononuclear cells were isolated from BM samples using lymphocyte separation medium (TBDscience Co., Ltd.) and total RNA was extracted using TRIzol ${ }^{\circledR}$ reagent (Invitrogen; Thermo Fisher Scientific, Inc.) according to the manufacturers' instructions. Complementary DNA was synthesized using the PrimeScript ${ }^{\mathrm{TM}}$ RT reagent Kit (Takara Biotechnology Co., Ltd.), and qPCR was conducted using iQTM SYBR ${ }^{\circledR}$ Green Supermix with the CFX384 Real-Time System (both Bio-Rad Laboratories, Inc.). GAPDH was used as the internal control. Melting curves were detected with only one peak and corresponded to the annealing temperature. The relative expression ratio was calculated using the $2^{-\triangle \triangle C q}$ method, and the primer sequences were as follows: EIF2S3 forward, 5'-ATCCCTGTCAACAGGAGGGA-3' and reverse, 5'-GGGGCCACATAAAAGGGAGAA-3'; and GAPDH forward, 5'TGTTGCCATCAATGACCCCTT-3' and reverse, 5-CTCCACGACGTACTCAGCG-3'.

Western blot analysis. Total protein was extracted using RIPA lysis buffer (Beyotime Institute of Biotechnology) and the concentration was determinated by the BCA method. Then approximately 25 ug proteins were boiled at $100{ }^{\circ} \mathrm{C}$ for 5 min and separated on $10 \%$ SDS-PAGE gels (Bio-Rad Laboratories, Inc.). The proteins were transferred to PVDF membranes (EMD Millipore), which were then blocked with $5 \%$ non-fat milk in TBST for $1 \mathrm{~h}$ at room temperature, and subsequently incubated overnight with antibodies against EIF2S3, AKT, p-AKT, ERK, p-ERK, p38, JNK and p-JNK (all Cell signaling Technology, Inc.). GAPDH (TransGen Biotech Co., Ltd.) was used as the loading control. The membranes were washed and then incubated with horseradish peroxidase-conjugated anti-mouse IgG or anti-rabbit IgG (TransGen Biotech Co., Ltd.) for $1 \mathrm{~h}$ at room temperature. The protein bands were visualized with ECL solution (Beyotime Institute of Biotechnology).

Cellular proliferation assay. The Cell Counting Kit-8 (CCK-8; TransGen Biotech Co., Ltd.) was used to assess the cellular proliferation rate, according to the manufacturer's instructions. The cells were seed into 96 -well plates ( $1 \times 10^{3}$ cells/well) and assessed at the indicated timepoints $(0,24,48,72,96,120$ and $144 \mathrm{~h}$ post-culture). The absorbance was measured at $450 \mathrm{~nm}$ and the proliferation rates were calculated. 
Cellular invasion assay. A cell invasion assay was performed using 8- $\mu$ m pore size Transwell plates (Costar; Corning, Inc.) precoated with Matrigel at a dilution 1:9 (BD Biosciences). A total of $1 \times 10^{4}$ cells/well in $200 \mu$ serum-free medium were seeded into the upper chambers, while the bottom chamber was filled with $500 \mu$ l complete culture medium (10\% FBS). After incubation for $24 \mathrm{~h}$ at $37^{\circ} \mathrm{C}$, the numbers of migratory cells in the bottom chamber were counted under a microscope.

Flow cytometric analysis. For cell cycle analysis, cells were washed twice with PBS and then fixed with cold $70 \%$ ethanol at $4{ }^{\circ} \mathrm{C}$ overnight. The fixed cells were then centrifuged, stained with propidium iodide (PI) solution ( $50 \mu \mathrm{g} / \mathrm{ml} ; 100 \mu \mathrm{g} / \mathrm{ml} \mathrm{RNase} \mathrm{A;} \mathrm{and} \mathrm{0.5 \%} \mathrm{Triton} \mathrm{X-100)} \mathrm{for}$ 30 min at $37^{\circ} \mathrm{C}$ in the dark. The stained cells were analyzed using a FACSCalibur flow cytometer (Becton, Dickinson and Company) and Flowjo software.

Mouse human tumor xenograft model. Following sodium pentobarbital anesthesia (50 mg/kg), HL-60-EIF2S3 cells (1x10 6 cells/100ul/mouse) were subcutaneously transplanted into 4-6-week-old BALB/C nude mice (Animal experimental center, Shanghai, China). The mice were divided into two groups ( $\mathrm{n}=6$ each); one group was fed with standard water and the other was administered water supplemented with DOX $(100 \mathrm{ng} / \mathrm{ml})$. Tumor volumes were evaluated every day using the following formula: Tumor volume (mm $\left.{ }^{3}\right)=$ (length $\mathrm{x}$ width $\left.{ }^{2}\right) / 2$. The mice were euthanized by $\mathrm{CO}_{2}$ inhalation after 18 days, and the volume of the cage displaced per minute was $20 \%$. The tumors were then removed and weighed. The study was performed according to the guidelines approved by the Animal Experimentation Ethics Committee of The First Affiliated Hospital of Guangzhou Medical University. The experiments were approved on December 10, 2019 and performed between January 2020 and April 2020.

Next-generation sequencing (NGS). Cells from the experimental group [HL60-EIF2S3 DOX (-)] and the control group [HL60-EIF2S3 DOX (+)] were assessed using mRNA NGS (Sinotech Genomics). And the data was uploaded to GEO database (GSE163683). The R package Edge was used to identify differentially expressed genes (DEGs) between the cells overexpressing EIF2S3 and the control cells. The cut-off criteria were $P$ value $<0.05$ and $\mid \log _{2}$ fold change (FC) $\mid \geq 2$. Gene Ontology (GO) function and Kyoto Encyclopedia of Genes and Genomes (KEGG) pathway enrichment analyses were performed on the identified DEGs.

Bioinformatics analysis. RNA-seq data from patients with AML were acquired from The Cancer Genome Atlas (TCGA) database, as well as clinical information including sex, age and FAB classification. The associations between EIF2S3 expression, clinical characteristics and AML prognosis were evaluated using UALCAN (http://ualcan.path.uab.edu/) and OncoLnc(http://www.oncolnc.org/).

Statistical analysis. All data were analyzed using GraphPad Prism 6.0 (GraphPad Software, Inc.) and SPSS 22.0 (IBM Corp). The experiments were repeated three times and are presented as the mean \pm standard deviation $(X \pm S D)$. Differences between the means of two or more experimental groups were assessed using the Mann-Whitney test, t-test or the analysis of variance (ANOVA) test. The log-rank test was used to assess the statistical significance of the Kaplan-Meier survival plots. Univariate and multivariate analyses were based on the Cox proportional hazards regression model. $\mathrm{P}<0.05$ was considered to indicate a statistically significant difference.

\section{Results}

TCGA bioinformatics analysis of the role of EIF2S3 in patients with AML. UALCAN was used to assess EIF2S3 mRNA expression levels in AML patients with different clinical characteristics. The results indicated that EIF2S3 expression may by associated with sex, age and FAB type. (Fig. S1). However, there were no obvious differences in EIF2S3 expression between different races or genetic abnormities (FLT3 mutation, PML-RAR gene fusion and Ras activation; Fig. S2). Furthermore, OncoLnc we used to evaluate the prognostic value of EIF2S3 in patients with AML from TCGA database. The results demonstrated that low EIF2S3 expression was significantly associated with poor prognosis ( $\mathrm{P}=0.003$; Fig. 1B).

Expression levels of EIF2S3 in patients with primary AML and CR. EIF2S3 mRNA expression levels were evaluated by RT-qPCR. A total of 61 AML samples were collected for experimentation. The results indicated that the expression levels of EIF2S3 in CR patients ( $n=20$ ) were higher than those in newly diagnosed patients $(n=41)(P=0.020)$ (Fig.1A).

Association between EIF2S3 expression and clinicopathological parameters. To further evaluate the impact of EIF2S3 in AML, the association between EIF2S3 expression and patient clinicopathological parameters was investigated. In total, 41 primary AML patient samples were divided into two groups according to the expression levels of EIF2S3 mRNA. High EIF2S3 expression was detected in 21 samples (51.22\%) and low expression was detected in 20 samples (48.78\%; Table 1). EIF2S3 expression was found to be correlated with patient $C R(P=0.012)$ and the outcome at study cut-off, whether alive or dead $(P=0.029)$. However, no significant association with patient age, sex, white blood cell (WBC) count, hemoglobin (HGB) level, platelet (PLT) count, blasts in the BM, FAB type or receipt of treatment was determined (Table 1). 
Association between EIF2S3 expression and prognosis in AML. Kaplan-Meier survival analysis demonstrated that high EIF2S3 expression resulted in increased overall survival (OS; $P=0.023$ ) and relapse-free survival (RFS; $P=0.013$ ) times (Fig. $1 C$ and $D)$. Furthermore, multivariate Cox regression analysis illustrated that EIF2S3 was an independent prognostic factor for OS $(P=0.013)$ and RFS $(P=0.008)$ (Table 2). In addition, univariate survival analysis indicated that age $(P=0.021)$ and treatment $(P=0.030)$ were also associated with $R F S$ in patients with AML (Table2).

EIF2S3 overexpression suppresses the proliferation of HEL and HL-60 cells. Western blotting was used to determine the EIF2S3 protein levels in AML and acute lymphocytic leukemia (ALL) cell lines, including THP-1, HEL, HL-60 and Hut102 cells (Fig. 2A). HEL and HL-60 cells overexpressing EIF2S3 were generated by inducible retrovirus infection. Empty vector-transfected cells were used as the control. Successful generation of HEL-EIF2S3, HEL control, HL-60-EIF2S3 and HL-60 control cells was confirmed by western blotting (Fig. 2B). In the overexpressing cells, EIF2S3 expression was suppressed in the presence of DOX. CCK-8 assays were performed to detect cellular proliferation, which revealed that upregulated EIF2S3 expression in HEL and HL-60 cells markedly suppressed proliferation rate compared with the control cells (Fig. 2C and D). The effects of the EIF2S3 gene on invasiveness were also evaluated, but no significant differences were observed between DOX (-) and DOX (+) cells (Fig. 2E and F).

EIF2S3 regulates the cell cycle in HEL and HL-60 cells. To further confirm the function of EIF2S3-mediated proliferation, flow cytometry was used to evaluate the effects of EIF2S3 overexpression on the cell cycle. The ratio of HEL-EIF2S3 DOX (-) to HL-60-EIF2S3 DOX (-) cells in the $G_{0 / 1}$ phase was significantly increased compared with the DOX (+) and control cells (Fig. $2 \mathrm{G}$ and $H$ ). By contrast, the number of HELEIF2S3 DOX (-) cells in the $G_{2} / M$ phase was decreased (Fig. 2G), as was the number of HL-60-EIF2S3 DOX (-) cells in the $S$ and $G_{2} / M$ phases (Fig. 2H). There was no significant difference between the control and DOX (+) cells. These results indicated that upregulated EIF2S3 expression resulted in $G_{0 / 1}$ cell cycle arrest and inhibited $G_{1} / S$ transition in $A M L$ cells.

Upregulation of EIF2S3 inhibits the tumorigenicity of AML in vivo. Considering that EIF2S3 overexpression inhibited AML cell proliferation in vitro, its influence on tumor formation was subsequently investigated in vivo. Due to ethics considerations and the results of the aforementioned assays (showing that DOX did not influence the experimental results), 12 mice were then divided into 2 groups, one group mice inoculated with HL-60 EIF2S3 cells, and the other group mice receiving water supplemented with DOX (100 ng/ml) as the control. Consistent with the proliferation assay results, tumors with excessive EIF2S3 expression grew significantly slower than those of the control animals one week after xenografting ( $\mathrm{P}=0.004$; Fig. $3 \mathrm{~B}$ ). After a further 18 days, the tumors were removed and the tumor weight and size recorded (Fig. 3 A). The mean tumor weight and size were lower in mice with EIF2S3 overexpression compared with the control mice $(P=0.015$; Fig. 3 C). Therefore, the results suggested that EIF2S3 may play an important role in the inhibition of acute leukemia cell growth in vivo.

Identification of DEGs, GO functional analysis and KEGG pathway enrichment. To investigate the mechanisms underlying the proliferative inhibition of HEL-EIF2S3 and HL-60-EIF2S3 cells, mRNA NGS was conducted using HL60-EIF2S3 DOX (-) and HL60-EIF2S3 DOX (+) cells (GSE163683). In total, 479 DEGs (including 271 upregulated and 208 downregulated genes) were identified from NGS, using P<0.05 and $\left|\log _{2} \mathrm{FC}\right| \geq 2$ as the cut-off criteria. A volcano plot of DEGs and heatmap of the top 30 DEGs are presented in Fig. S3 A and B. GO functional analysis highlights relevant biological processes associated with DEGs, which showed that the identified DEGs were primarily involved in 'leukocyte homeostasis', 'lymphocyte homeostasis' and 'execution phase of apoptosis' (Fig. S4). KEGG pathway enrichment analysis identified the relevant signaling pathways associated with these DEGs. In order of the number of DEGs involved in the pathway, the results revealed that the second most enriched pathway was the MAPK signaling pathway, which is a common tumor signaling pathway (Fig. 4). After filtering the pathways (Fig. 4), the DEGs involved in five common cell signaling pathways (including those of MAPK, Ras, PI3K-Akt, FoxO and Rap1) were further investigated (heatmap, Fig 5). Furthermore, the top 30 enriched pathways also revealed that the DEGs were primarily enriched in the MAPK pathway (based on the rich factor; Fig. S5). Combining the number of DEGs involved in the pathways and the rich factor, these results suggested that the function of EIF2S3 may be associated with MAPK signaling. The expression of 15 DEGs in the MAPK signaling pathway was then confirmed by RT-qPCR, and the expression of 11 genes was markedly different between EIF2S3 overexpressing cells and the controls, which verified the reliability of the sequencing results (Fig. S6).

Relevant proteins and their phosphorylation levels in the MAPK signaling pathway. The results of NGS revealed that EIF2S3 may inhibit AML cell proliferation and $G_{1} / S$ transition via the MAPK signaling pathway. Western blotting was used to detect relevant pathway proteins and their phosphorylation levels in AML cells, including ERK, p-ERK, P38, p-P38, JNK1/2/3 and p-JNK1/2/3. The results showed that the levels of p-ERK were notably decreased in HEL-EIF2S3 DOX (-) and HL-60-EIF2S3 DOX (-) cells compared with their DOX (+) counterparts (Fig. 6; P囚0.001). Collectively, the results suggested that differences in EIF2S3 expression altered the levels of ERK phosphorylation in AML cells, the core protein in the MAPK/ERK signaling pathway. 


\section{Discussion}

AML is a common hematological malignancy with high incidence and mortality rates (19). With developments in cytogenetics and molecular biology, chromosomal translocations in AML have been revealed, including $t(8 ; 21)$ (q22;q22), inv(16)(p13q22b) and t(16;16) (p13;q22). Gene fusions have also been discovered (such as BCR-ABL1, MLLT1-ETO and CBFß-MYH11), as well as mutations in the nucleophosmin, NRas and runt-related transcription factor 1 genes (20). These alterations were significantly correlated with clinical prognosis. As such, various novel molecular targeted therapies have emerged, improving on traditional treatments such as chemotherapy and hematopoietic stem cell transplantation.

With its GTP binding domain, EIF2S3 is one of the core subunits of EIF2, and plays an important role in eukaryotic translation initiation (69). However, relevant studies surrounding the function and mechanism of EIF2S3 in AML are limited. In the present study, data from TCGA database was used to evaluate the role of EIF2S3 in AML. The results indicated that EIF2S3 expression may by associated with sex, age and $F A B$ type. Existing studies have suggested that $A M L$ is a disease in which age is closely associated with incidence and cure rate, and that the cure rates are lower in older patients (21). Combined with this phenomenon, EIF2S3 expression was lower in 81-100-year-old patients, which indicated that it may be associated with poor outcome. Survival analysis using OncoLnc showed that the high-EIF2S3 expression group exhibited improved prognosis compared with the low-expression group, suggesting that EIF2S3 may be a potential prognostic factor for AML.

To further verify these conclusions, $41 \mathrm{BM}$ specimens from patients with primary AML, and 20 specimens from patients with CR, were collected. The results demonstrated that EIF2S3 expression levels were higher in patients with CR than in those with primary AML. Furthermore, there were no significant differences in EIF2S3 expression by age, sex, WBC count, HGB level, PLT count, blasts in the BM, FAB type or treatment. This was not consistent with the conclusions from previous bioinformatics analyses, which was potentially the result of the limited number of enrolled patients in the present study. However, the results showed a significant association between EIF2S3 expression, remission status and treatment outcome. The enrolled patients were followed up for 71 months and survival analysis illustrated that EIF2S3 was an independent prognostic factor for OS and RFS. To conclude, EIF2S3 may be a favorable indicator of disease status and prognosis in AML.

Subsequently, the function and mechanism of EIF2S3 in AML were investigated using in vivo and invitro experimentation. In vitro, the Retro-X Tet-Off Advanced inducible expression system was used to generate AML cells overexpressing EIF2S3 (HEL-EIF2S3 and HL-60 EIF2S3 cells). EIF2S3 overexpression suppressed the proliferation rates and inhibited $G_{0 / 1}$ to $S$ phase transition in AML cells. Moreover, EIF2S3 inhibited the tumorigenicity of HL-60 cells in mice models. To investigate the mechanisms of EIF2S3 in AML, mRNA NGS with HL60-EIF2S3 DOX (-) and HL60-EIF2S3 DOX (+) cells highlighted 479 DEGs (including 271 upregulated and 208 downregulated genes). GO functional analysis showed that these DEGs were primarily involved in the biological functions 'leukocyte homeostasis', 'lymphocyte homeostasis' and 'execution phase of apoptosis', suggesting that EIF3S3 affects AML via these processes. In order of the number of DEGs involved in the signaling pathway, KEGG pathway enrichment analysis revealed that the second most enriched pathway was the MAPK signaling pathway, which is commonly associated with tumor formation. This indicates that the function of EIF2S3 may be associated with the MAPK signaling pathway. In addition, western blot analysis revealed that the protein levels of $p$-ERK were markedly decreased in HEL-EIF2S3 DOX (-) and HL-60-EIF2S3 DOX (-) cells compared with the associated controls. The differences in EIF2S3 expression in AML cells were found to alter the phosphorylation levels of ERK, which is one of the fundamental proteins in the MAPK/ERK signaling pathway.

Eukaryotic cells receive external signals which are then transmitted to critical targets within the cell to achieve the appropriate biological response. These signal transduction processes are often initiated by receptor tyrosine kinases (RTKs); an essential effector cascade required for most RTK functions is the MAPK cascade, comprised of the Raf, MEK and ERK kinases. The ERK1/2 signaling pathway has been widely researched $(22,23)$. Previous studies have demonstrated that ERK1/2 signaling is closely associated with cellular proliferation, the cell cycle and apoptosis (24). ERK activation induces the cell cycle and inhibits negative regulators of the cell cycle, which is necessary for $G_{1} / S$ transition (25). ERK activation also allowed successful $G_{1}$ phase progression by inducing the assembly of cyclin $D$ and the cyclin D-CDK4 complex, mediating Myc protein stability, regulating the expression of p21 and p27 and downregulating the expression of antiproliferative genes (26-27). In leukemia, the MAPK signaling pathway (including the ERK1/2 pathway) was found to be abnormally activated in progenitor cells and suppressing ERK activity inhibited the proliferation of primary acute myeloid leukemia cells and induced apoptosis (29). In summary, EIF2S3 overexpression may suppress the cellular proliferation and promote cell cycle arrest by inhibiting MAPK/ERK signal activation in AML.

To the best of our knowledge, the present study is the first to investigate the function and mechanism of EIF2S3 in leukemia; therefore, some deficiencies and limitations are worth reporting. First, according to the 2016 WHO classification, AML is classified by morphology, 
genetic abnormalities and chromosome translocation. However, genetic and chromosomal data from the enrolled patients were incomplete. Therefore, the association between EIF2S3 and genetic or chromosomal abnormalities could not be accurately analyzed, which may have affected the conclusions. Second, based on ethics, the comparison of EIF2S3 expression level in the bone marrow of healthy people and patients was lacking. the blood samples of patients and healthy people would be collected in the next stage of research to compare the expression level of EIF2S3. In addition, a limited number of patients were enrolled in the present study; therefore, more samples and clinical data must be collected in the future to confirm the findings of the present study.

In conclusion, EIF2S3 expression may inhibit the cell cycle, cellular proliferation and tumorigenicity in AML by inhibiting the activation of the MAPK/ERK signaling pathway. However, further research is required to determine the relevant underlying mechanisms.

\section{Declarations}

\section{Funding}

The present study was supported by the National Natural Science Foundation of China (grant no. 81672661, 81870113) and the Guangzhou Science and Technology Project (grant no. 201804010199).

\section{Conflicts of Interest}

The authors declare that they have no competing interests.

\section{Availability of data and materials}

The datasets used and/or analyzed during the current study are available from the corresponding author on reasonable request.

\section{Code availability}

Not applicable.

\section{Author contributions}

$L X, Y Z$ and $Z H$ conceived the study idea, supervised the project and designed the experiments; JL and SC performed the experiments and wrote the manuscript; $L L, H T, B L, Y C$ and $X Z$ collected the samples, performed the experiments and analyzed the data.

\section{Ethics approval and consent to participate}

The present study was approved by the ethics committee of the First Affiliated Hospital of Guangzhou Medical University. All patients provided written informed consent for the use of their clinical specimens. Animals were purchased from the Animal experimental center, Shanghai, China, and the protocol was approved by the Institutional Animal Care and Use Committee of Guangzhou Medical University. All mouse procedures were performed in strict accordance with the recommendations of the Guide for the Care and Use of Laboratory Animals of Guangzhou Medical University. Animal experiments were performed at Guangzhou Medical University and all surgeries were performed under sodium pentobarbital anesthesia; all efforts were made to minimize animal suffering.

\section{Acknowledgements}

Not applicable.

\section{References}

1. Narayanan D, Weinberg OK. How I investigate acute myeloid leukemia. Int J Lab Hematol. 2020;42(1):3-15. https://doi.org/10.1111/ijlh.13135

2. Siegel RL, Miller KD, Jemal A. Cancer statistics, 2019. CA: a cancer journal for clinicians. 2019;69(1):7-34. https://doi.org/10.3322/caac. 21551

3. Chen W, Zheng R, Baade PD, et al. Cancer statistics in China, 2015. CA Cancer J Clin. 2016;66(2):115-132. https://doi.org/10.3322/caac.21338

4. Coombs CC, Tallman MS, Levine RL. Molecular therapy for acute myeloid leukaemia. Nature reviews Clinical oncology. 2016;13(5):305-318. https://doi.org/10.1038/nrclinonc.2015.210 
5. Bose P, Vachhani P, Cortes JE. Treatment of Relapsed/Refractory Acute Myeloid Leukemia. Curr Treat Options Oncol. 2017;18(3):17. https://doi.org/10.1007/s11864-017-0456-2

6. Hinnebusch AG. The scanning mechanism of eukaryotic translation initiation. Annual review of biochemistry. 2014;83:779-812. https://doi.org/10.1146/annurev-biochem-060713-035802

7. Hinnebusch AG. Molecular mechanism of scanning and start codon selection in eukaryotes. Microbiol Mol Biol Rev. 2011;75(3):434467, first page of table of contents. https://doi.org/ 10.1128/MMBR.00008-11

8. Hinnebusch AG, Lorsch JR. The mechanism of eukaryotic translation initiation: new insights and challenges. Cold Spring Harbor perspectives in biology. 2012;4(10). https://doi.org/ 10.1101/cshperspect.a011544

9. Young-Baird SK, Shin BS, Dever TE. MEHMO syndrome mutation EIF2S3-I259M impairs initiator Met-tRNAiMet binding to eukaryotic translation initiation factor elF2. Nucleic Acids Res. 2019;47(2):855-867. https://doi.org/ 10.1093/nar/gky1213

10. Stanik J, Skopkova M, Stanikova D, et al. Neonatal hypoglycemia, early-onset diabetes and hypopituitarism due to the mutation in EIF2S3 gene causing MEHMO syndrome. Physiol Res. 2018;67(2):331-337. https://doi.org/ 10.33549/physiolres.933689. Epub 2018 Jan 5.

11. Skopkova M, Hennig F, Shin BS, et al. EIF2S3 Mutations Associated with Severe X-Linked Intellectual Disability Syndrome MEHMO. Hum Mutat. 2017;38(4):409-425. https://doi.org/ 10.1002/humu.23170. Epub 2017 Jan 23.

12. Moortgat S, Desir J, Benoit V, et al. Two novel EIF2S3 mutations associated with syndromic intellectual disability with severe microcephaly, growth retardation, and epilepsy. American journal of medical genetics Part A. 2016;170(11):2927-2933. https://doi.org/10.1002/ajmg.a.37792

13. Borck G, Shin BS, Stiller B, et al. elF2gamma mutation that disrupts elF2 complex integrity links intellectual disability to impaired translation initiation. Molecular cell. 2012;48(4):641-646. https://doi.org/ 10.1016/j.molcel.2012.09.005

14. Chian CF, Hwang YT, Terng HJ, et al. Panels of tumor-derived RNA markers in peripheral blood of patients with non-small cell lung cancer: their dependence on age, gender and clinical stages. Oncotarget. 2016;7(31):50582-50595. https://doi.org/10.18632/oncotarget.10558

15. Chang YT, Huang CS, Yao CT, et al. Gene expression profile of peripheral blood in colorectal cancer. World J Gastroenterol. 2014;20(39):14463-14471. https://doi.org/ 10.3748/wjg.v20.i39.14463

16. Dohner H, Estey E, Grimwade D, et al. Diagnosis and management of AML in adults: 2017 ELN recommendations from an international expert panel. Blood. 2017;129(4):424-447. https://doi.org/

17. Arber DA, Orazi A, Hasserjian R, et al. The 2016 revision to the World Health Organization classification of myeloid neoplasms and acute leukemia. Blood. 2016;127(20):2391-2405. https://doi.org/ 10.1182/blood-2016-08-733196

18. Dohner H, Estey EH, Amadori S, et al. Diagnosis and management of acute myeloid leukemia in adults: recommendations from an international expert panel, on behalf of the European LeukemiaNet. Blood. 2010;115(3):453-474. https://doi.org/ 10.1182/blood-200907-235358

19. Siegel RL, Miller KD, Jemal A. Cancer statistics, 2020. CA Cancer J Clin. 2020;70(1):7-30. https://doi.org/10.3322/caac.21590

20. Grimwade D, Hills RK, Moorman AV, et al. Refinement of cytogenetic classification in acute myeloid leukemia: determination of prognostic significance of rare recurring chromosomal abnormalities among 5876 younger adult patients treated in the United Kingdom Medical Research Council trials. Blood. 2010;116(3):354-365. https://doi.org/ 10.1182/blood-2009-11-254441

21. Dores GM, Devesa SS, Curtis RE, Linet MS, Morton LM. Acute leukemia incidence and patient survival among children and adults in the United States, 2001-2007. Blood. 2012;119(1):34-43. https://doi.org/ 10.1182/blood-2011-04-347872

22. Lavoie H, Therrien M. Regulation of RAF protein kinases in ERK signalling. Nature reviews Molecular cell biology. 2015;16(5):281-298. https://doi.org/ 10.1038/nrm3979.

23. Guo YJ, Pan WW, Liu SB, Shen ZF, Xu Y, Hu LL. ERK/MAPK signalling pathway and tumorigenesis. Exp Ther Med. 2020;19(3):19972007. https://doi.org/ 10.3892/etm.2020.8454

24. Saez-Rodriguez J, MacNamara A, Cook S. Modeling Signaling Networks to Advance New Cancer Therapies. Annu Rev Biomed Eng. 2015;17:143-163. https://doi.org/ 10.1146/annurev-bioeng-071813-104927

25. Yamamoto T, Ebisuya M, Ashida F, Okamoto K, Yonehara S, Nishida E. Continuous ERK activation downregulates antiproliferative genes throughout G1 phase to allow cell-cycle progression. Current biology : CB. 2006;16(12):1171-1182. https://doi.org/ 10.1016/j.cub.2006.04.044

26. Meloche S, Pouyssegur J. The ERK1/2 mitogen-activated protein kinase pathway as a master regulator of the G1- to S-phase transition. Oncogene. 2007;26(22):3227-3239. https://doi.org/ 10.1038/sj.onc.1210414

Page $8 / 16$ 
27. Vasjari L, Bresan S, Biskup C, Pai G, Rubio I. Ras signals principally via Erk in G1 but cooperates with PI3K/Akt for Cyclin D induction and S-phase entry. Cell Cycle. 2019;18(2):204-225. https://doi.org/ 10.1080/15384101.2018.1560205

28. Lee JT, Jr., McCubrey JA. The Raf/MEK/ERK signal transduction cascade as a target for chemotherapeutic intervention in leukemia. Leukemia. 2002;16(4):486-507. https://doi.org/ 10.1038/sj.leu.2402460

29. Su Y, Li X, Ma J, et al. Targeting PI3K, mTOR, ERK, and Bcl-2 signaling network shows superior antileukemic activity against AML ex vivo. Biochem Pharmacol. 2018;148:13-26. https://doi.org/ 10.1016/j.bcp.2017.11.022

\section{Tables}


Table 1

Association between EIF2S3 expression and clinicopathological parameters in 41 primary AML patients

\begin{tabular}{|c|c|c|c|c|}
\hline \multirow[t]{2}{*}{ Clinicopathological variables } & \multirow[t]{2}{*}{ Cases (41) } & \multicolumn{2}{|c|}{ EIF2S3 expression value } & \multirow[t]{2}{*}{$P$ value } \\
\hline & & low (20) & High (21) & \\
\hline \multicolumn{5}{|l|}{ Age (years) } \\
\hline$\leq 60$ & 31 & 15 & 16 & 1.000 \\
\hline$>60$ & 10 & 5 & 5 & \\
\hline \multicolumn{5}{|l|}{ Gender } \\
\hline Male & 18 & 8 & 10 & 0.756 \\
\hline Female & 23 & 12 & 11 & \\
\hline \multicolumn{5}{|l|}{$\mathrm{WBC}^{\mathrm{a})}(\times 109 / \mathrm{L})$} \\
\hline$<10$ & 19 & 8 & 11 & 0.536 \\
\hline$\geq 10$ & 22 & 13 & 9 & \\
\hline \multicolumn{5}{|l|}{$\mathrm{HGB}^{\mathbf{b})}(\mathrm{g} / \mathrm{L})$} \\
\hline$<80$ & 21 & 13 & 8 & 0.121 \\
\hline$\geq 80$ & 20 & 7 & 13 & \\
\hline \multicolumn{5}{|l|}{$\left.\mathrm{PLT}^{c}\right)(\times 109 / \mathrm{L})$} \\
\hline$<50$ & 22 & 9 & 13 & 0.354 \\
\hline$\geq 50$ & 19 & 11 & 8 & \\
\hline \multicolumn{5}{|l|}{ Blast in $B M^{d)}$} \\
\hline$<50 \%$ & 14 & 6 & 8 & 0.737 \\
\hline$\geq 50 \%$ & 24 & 13 & 11 & \\
\hline \multicolumn{5}{|l|}{ FAB type } \\
\hline MO & 1 & 1 & 0 & 0.132 \\
\hline M1 & 3 & 2 & 1 & \\
\hline M2 & 12 & 6 & 6 & \\
\hline M3 & 6 & 1 & 5 & \\
\hline M4 & 7 & 2 & 5 & \\
\hline M5 & 7 & 6 & 1 & \\
\hline M6 & 1 & 0 & 1 & \\
\hline \multicolumn{5}{|l|}{ Complete Remission ${ }^{\text {f) }}$} \\
\hline Yes & 19 & 5 & 14 & 0.012 \\
\hline No & 22 & 15 & 7 & \\
\hline \multicolumn{5}{|l|}{ Treatment } \\
\hline chemotherapy & 28 & 13 & 15 & 0.172 \\
\hline transplantation & 10 & 4 & 6 & \\
\hline none & 3 & 3 & 0 & \\
\hline Outcome at study cut-off & & & & 0.029 \\
\hline
\end{tabular}

Page $10 / 16$ 


\begin{tabular}{|llll|} 
Alive & 20 & 6 & 14 \\
\hline Dead & 21 & 14 & 7 \\
\hline
\end{tabular}

Note: ${ }^{*} P<0.05$, the difference was statistically significant.

Abbreviations: a) $W B C$ white blood cell, b) $H G B$ hemoglobin, c) PLT Platelets, d) Blast in BM blast percentage in bone marrow, f) $C R$ complete remission

Table 2

Cox-regression analysis of parameters associated with OS and RFS of AML patients

\begin{tabular}{|c|c|c|c|c|c|c|c|c|}
\hline \multirow[t]{3}{*}{ Factors } & \multirow{2}{*}{\multicolumn{2}{|c|}{$\begin{array}{l}\text { OS }{ }^{\text {a) }} \\
\text { U variate }\end{array}$}} & \multicolumn{6}{|c|}{$\left.\mathrm{RFS}^{b}\right)$} \\
\hline & & & \multicolumn{2}{|c|}{ Multivariate } & \multicolumn{2}{|l|}{ U variate } & \multicolumn{2}{|c|}{ Multivariate } \\
\hline & $P$ & $H R^{c)}$ & $\begin{array}{l}95 \% \\
\mathrm{Cl}\end{array}$ & $P$ & $P$ & $\mathrm{HR}$ & $95 \% \mathrm{Cl}$ & $P$ \\
\hline Age, years $(\leq 60 />60)$ & 0.059 & 2.921 & $\begin{array}{l}1.134- \\
7.526\end{array}$ & 0.026 & 0.021 & 3.260 & $\begin{array}{l}1.302- \\
8.160\end{array}$ & 0.012 \\
\hline Gender(male/female) & 0.743 & - & - & - & 0.887 & - & - & - \\
\hline WBC $^{\text {d) }}(<10 / \geq 1 \times 109 / L)$ & 0.862 & - & - & - & 0.994 & - & - & - \\
\hline $\mathrm{HGB}$ e) $(<80 / \geq 80, \mathrm{~g} / \mathrm{L})$ & 0.142 & - & - & - & 0.200 & - & - & - \\
\hline $\operatorname{PLT} f(<50 / \geq 50, \times 109 / L)$ & 0.198 & - & - & - & 0.123 & - & - & - \\
\hline Blast in $\mathrm{BM}$ g) $(<50 \% / \geq 50 \%)$ & 0.400 & - & - & - & 0.483 & - & - & - \\
\hline EIF2S3 expression (high/low) & 0.023 & 0.299 & $\begin{array}{l}0.115- \\
0.772\end{array}$ & 0.013 & 0.011 & 0.277 & $\begin{array}{l}0.108- \\
0.716\end{array}$ & 0.008 \\
\hline Treatment(chemotherapy/transplantation/none) & 0.132 & 0.618 & $\begin{array}{l}0.299- \\
1.281\end{array}$ & 0.196 & 0.030 & 0.717 & $\begin{array}{l}0.370- \\
1.387\end{array}$ & 1.387 \\
\hline
\end{tabular}

Note: ${ }^{*} P<0.05$, the difference was statistically significant.

Abbreviations: a) OS overall survival, b) RFS relapse-free survival, c) $H R$ hazard ratio, d) WBC white blood cell, e) $H G B$ hemoglobin, f) $P L T$ Platelets, g) Blast in BM blast percentage in bone marrow

\section{Figures}


A

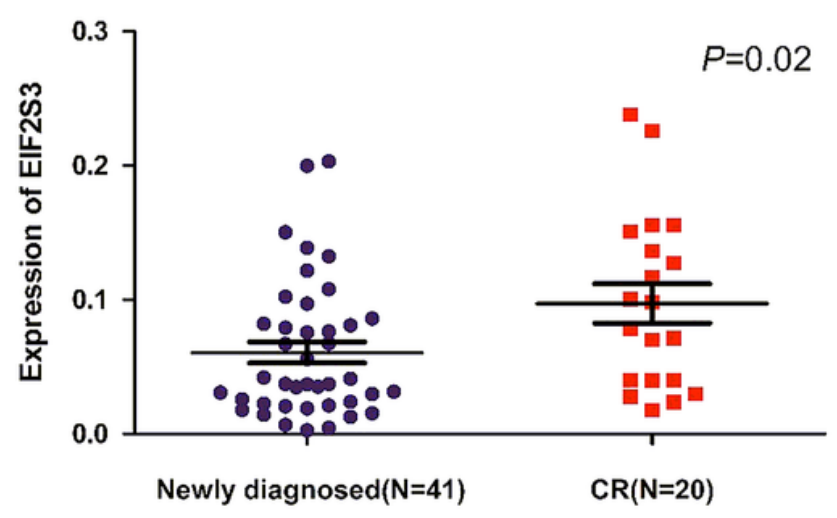

C

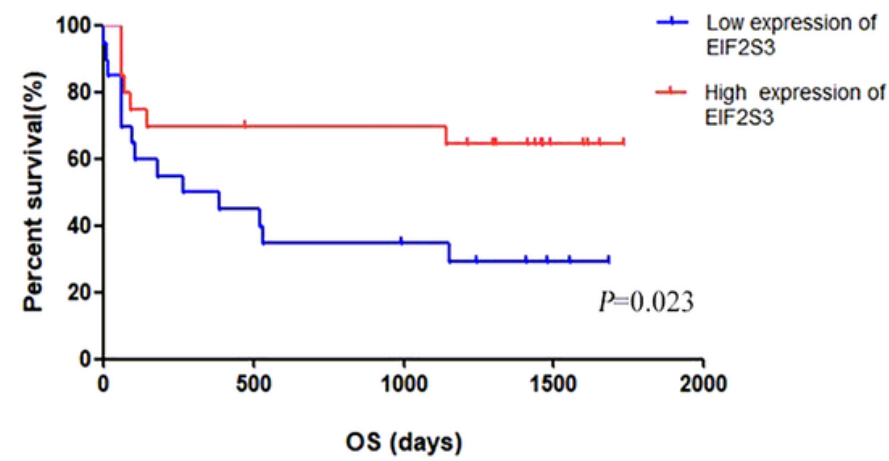

B

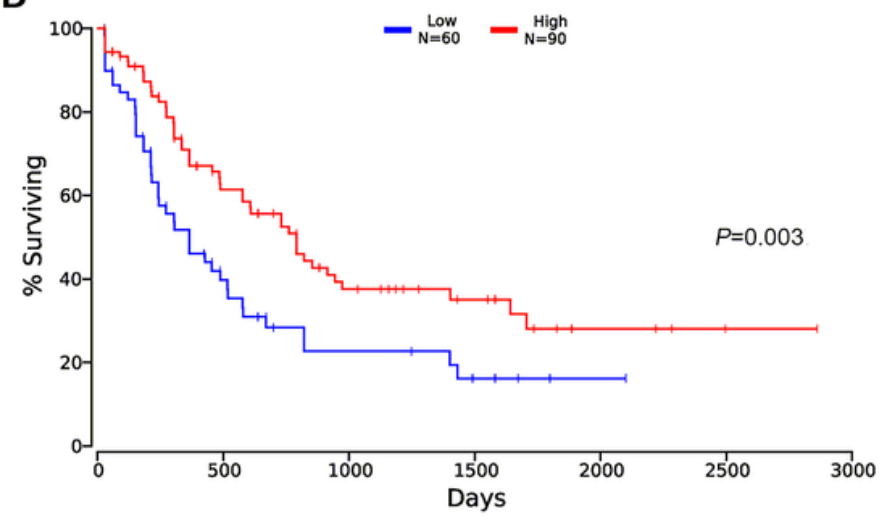

D

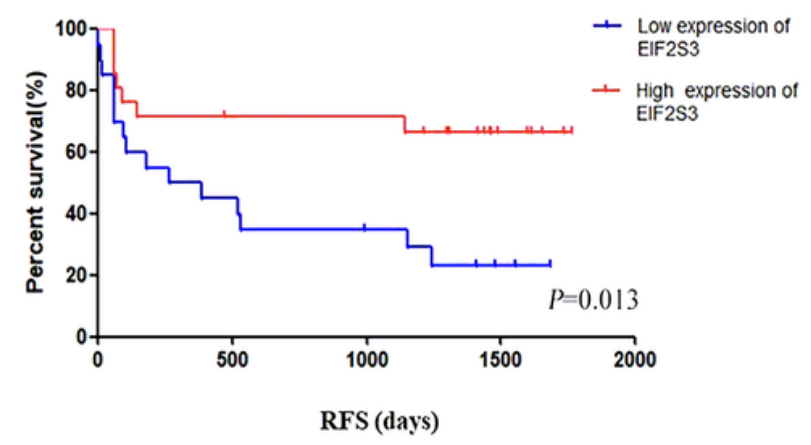

Figure 1

Association between EIF2S3 mRNA expression levels in patients with primary AML or CR and survival analysis. (A) A total of 61 samples were collected from patients with $A M L$, and reverse transcription-quantitative PCR was used to detect EIF2S3 mRNA expression in newlydiagnosed $(n=41)$ and $C R(n=20)$ patients $(P=0.02)$. (B) According to the differences in EIF2S3 expression level, patients from The Cancer Genome Atlas with the top $60 \%$ expression levels were defined as the high-expression group, and the remaining patients were classified as the low-expression group. Univariate survival analysis was used to investigate the prognostic effects of EIF2S3 in AML (P=0.0037). (C) Univariate survival analysis of OS in 41 enrolled patients with primary AML. High EIF2S3 expression was detected in 21 samples (51.22\%) and low EIF2S3 expression was detected in 20 samples (48.78\%) ( $P=0.023$ ). (D) Univariate survival analysis of RFS in 41 patients with primary AML $(P=0.013)$. EIF2S3, eukaryotic translation initiation factor 2 subunit 3; $A M L$, acute myeloid leukemia; $C R$, complete remission; OS, overall survival; RFS, relapse-free survival. 
A
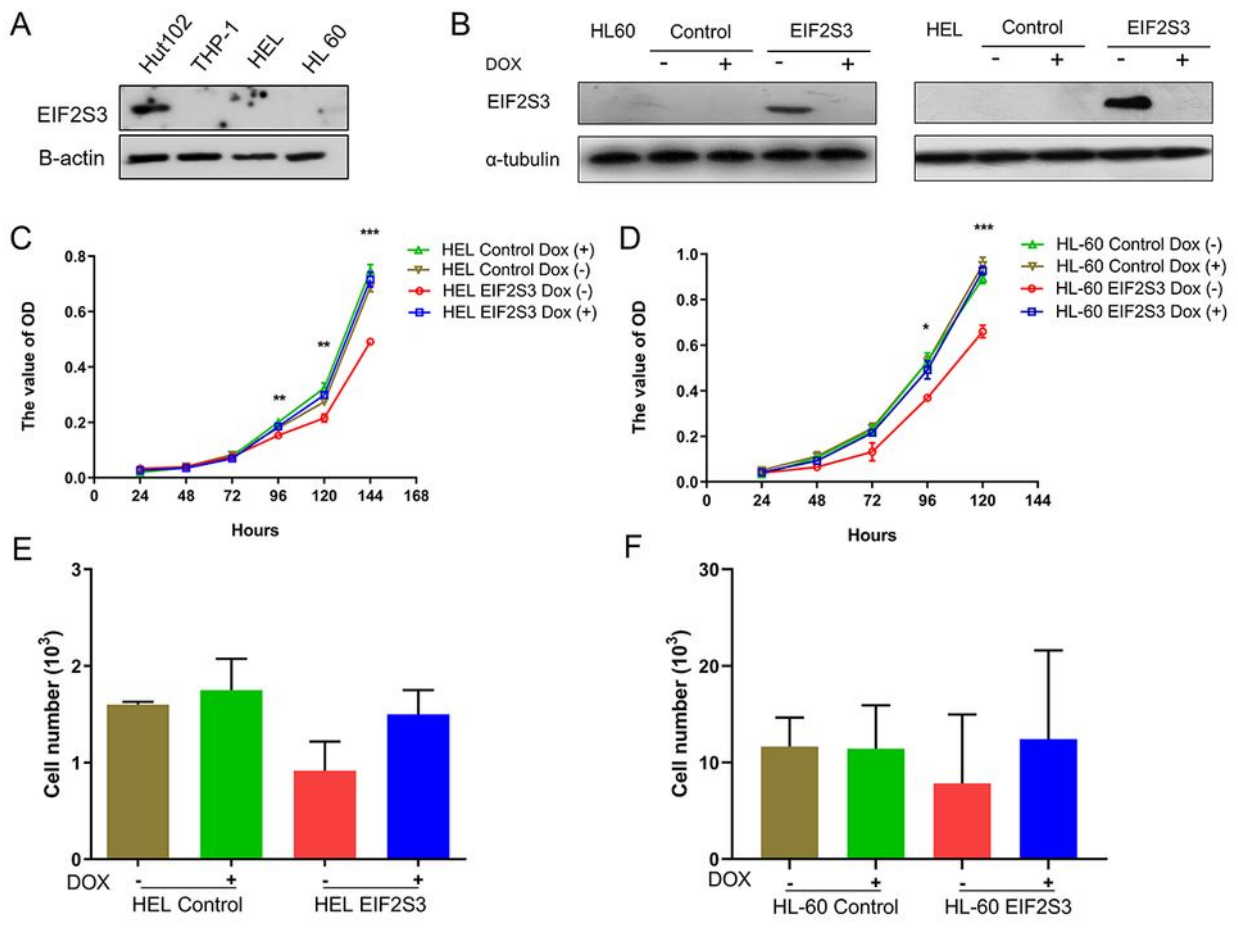

G
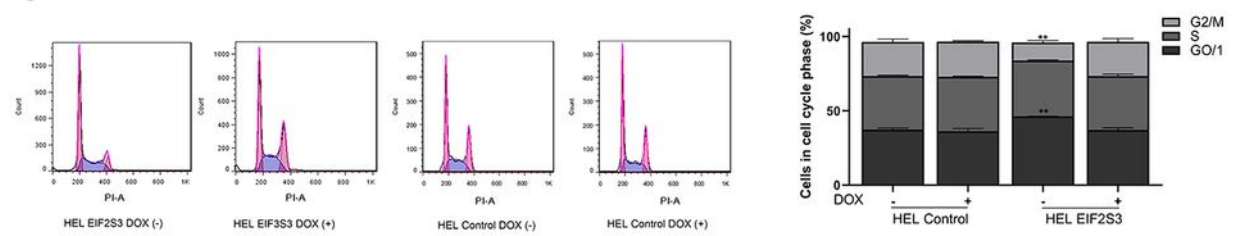

$\mathrm{H}$
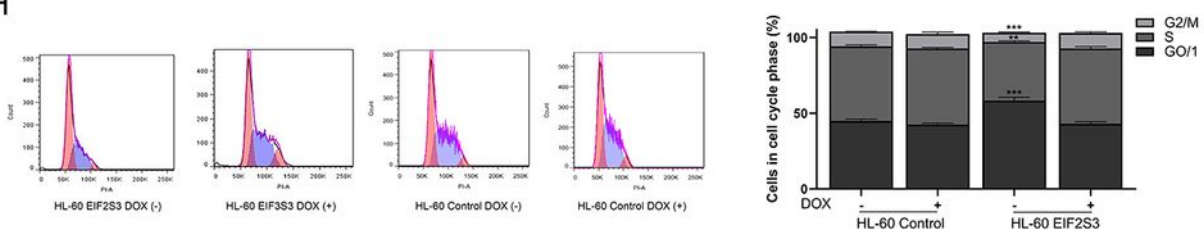

\section{Figure 2}

Overexpression of EIF2S3 suppresses cellular proliferation and G1/S transition in HL-60 and HEL cells. (A) Western blot analysis of EIF2S3 protein levels in AML (THP-1, HEL and HL-60) and ALL (Hut102) cell lines. (B) Western blotting was used to confirm the successful overexpression of EIF2S3 in AML and the control cells. (C) Proliferation of HEL-EIF2S3 and HEL control cells was assessed at 24, 48, 72, $96(P=0.001), 120(P=0.001)$ and $144 \mathrm{~h}(\mathrm{P}<0.001)$. (D) Proliferation of HL-60-EIF2S3 and HL-60 control cells was evaluated at 24, 48, 72 , $96(P=0.006)$ and $120 \mathrm{~h}(\mathrm{P}<0.001)$. ( $\mathrm{E}$ and $\mathrm{F})$ Cellular invasion analysis showed no significant differences between DOX $(-)$ and DOX (+) cells $(P>0.05)$. ( $G$ and $H)$ Flow cytometry was used to investigate the cell cycle distribution of HEL-EIF2S3, HEL control, HL-60-EIF2S3 and HL-60 control cells. Compared with HEL-EIF2S3 DOX (+) and control cells, HEL-EIF2S3 DOX (-) cells in the G0/1 phase $(P=0.001)$ were increased, and decreased in the G2/M phase ( $P=0.002)$. Compared with HL-60-EIF2S3 DOX (+) and the control cells, HL-60-EIF2S3 DOX (-) cells in the G0/1 $(P<0.001)$ and $S$ phase $(P=0.003)$ were increased, and those in the $G 2 / M$ phase $(P<0.001)$ were decreased. Results of three independent experiments are shown. Data are presented as the mean $\pm S E M . * P<0.05, * * P<0.01$ and $* \star * P<0.001$. EIF2S3, eukaryotic translation initiation factor 2 subunit 3; AML, acute myeloid leukemia; ALL, acute lymphocytic leukemia; DOX, doxycycline. 
A

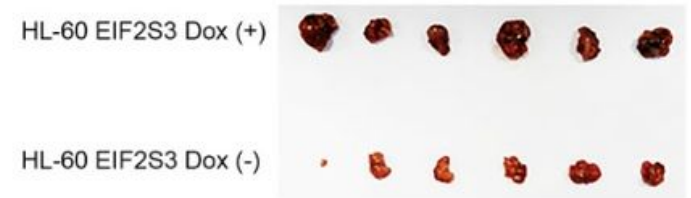

B

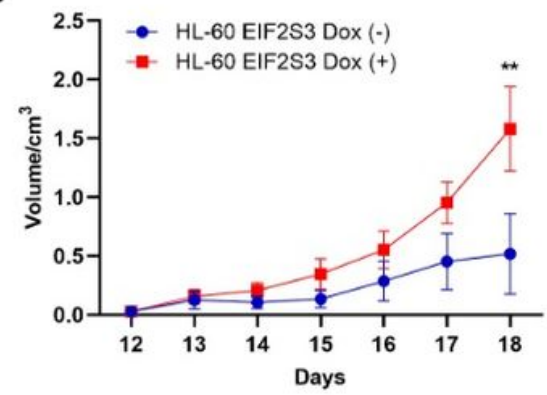

C

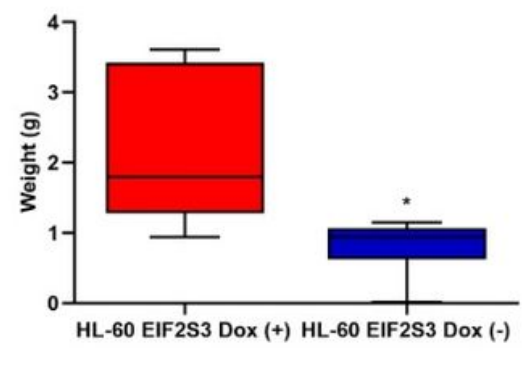

Figure 3

Upregulation of EIF2S3 expression inhibits the tumorigenicity of AML in model mice. (A) Tumors were removed from mice after inoculation for 18 days. (B) Growth curve $(P=0.004)$ and $(C)$ weight $(P=0.015)$ of xenografted tumors showed that EIF2S3 overexpression decreased tumor growth and size. EIF2S3, eukaryotic translation initiation factor 2 subunit 3; AML, acute myeloid leukemia; DOX, doxycycline.

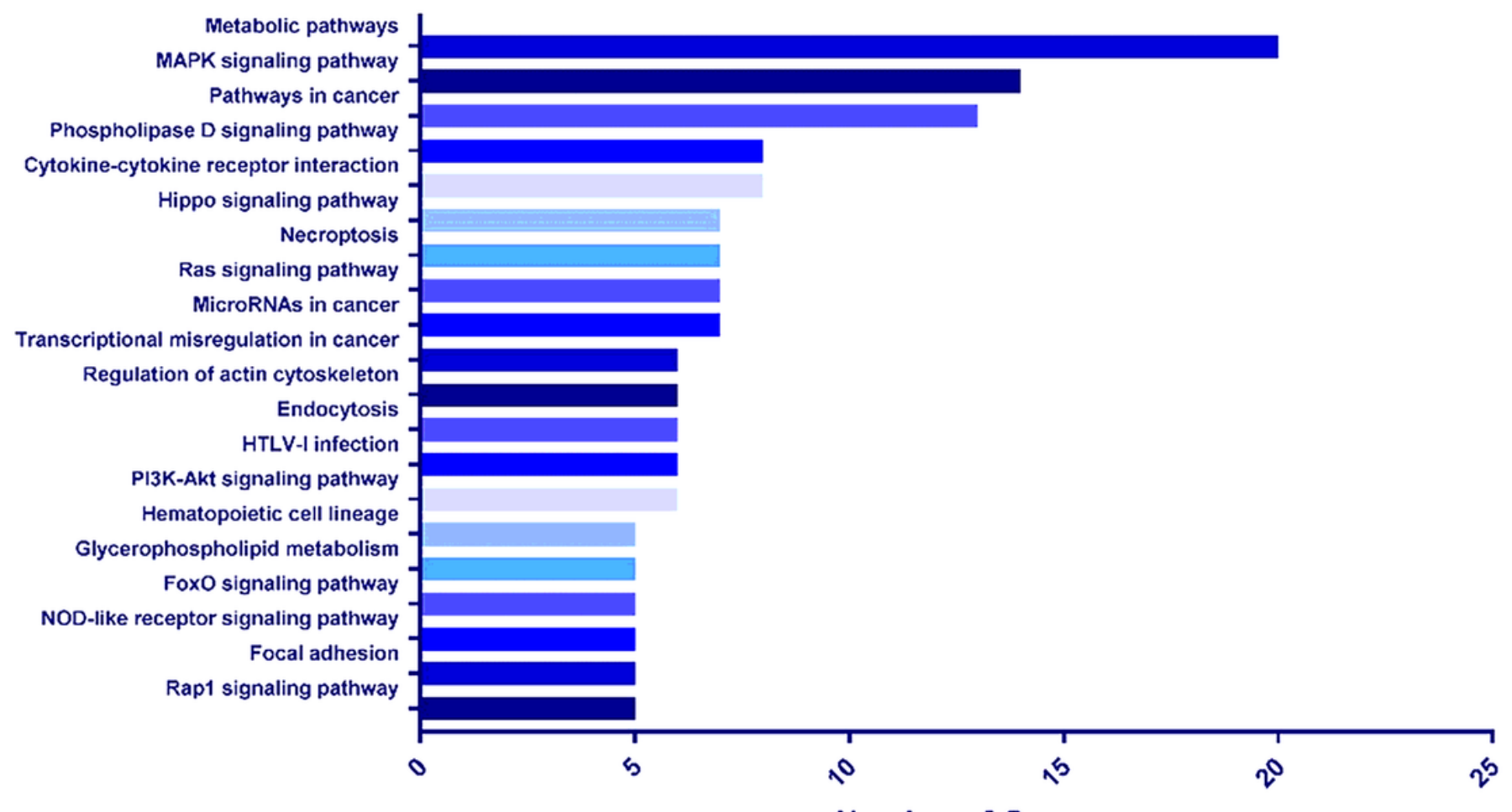

Number of Gene

Figure 4

Identification of KEGG enriched pathways in HL60-EIF2S3 DOX (-) and HL60-EIF2S3 DOX (+) cells by next-generation sequencing. KEGG pathway enrichment analysis described the relevant signaling pathways in order of number of DEGs. KEGG, Kyoto Encyclopedia of Genes and Genomes; DEG, differentially expressed genes; DOX, doxycycline. 
A

\begin{tabular}{|c|c|c|}
\hline $\begin{array}{c}\text { Signaling } \\
\text { pathway }\end{array}$ & NO. gene & Gene \\
\hline MAPK & 14 & $\begin{array}{r}\text { MAP3K13, CRK, MRAS, DDIT3, PLA2G4B, } \\
\text { DUSP3, KITLG, MAX, TGFBR1, STK4, JUND, } \\
\text { EFNA3, JMJD7-PLA2G4B }\end{array}$ \\
\hline Ras & 7 & $\begin{array}{r}\text { MRAS, PLA2G4B, CALML4, KITLG, STK4, EFNA3, } \\
\text { JMJD7-PLA2G4B }\end{array}$ \\
\hline PI3K-Akt & 6 & MYC, COL6A3, KITLG, CREB3L3, EFNA3, IL7R \\
\hline FoxO & 5 & PLK2, RAG1, TGFBR1, STK4, IL7R \\
\hline Rap1 & 5 & CRK, MRAS, CALML4, KITLG, EFNA3 \\
\hline
\end{tabular}

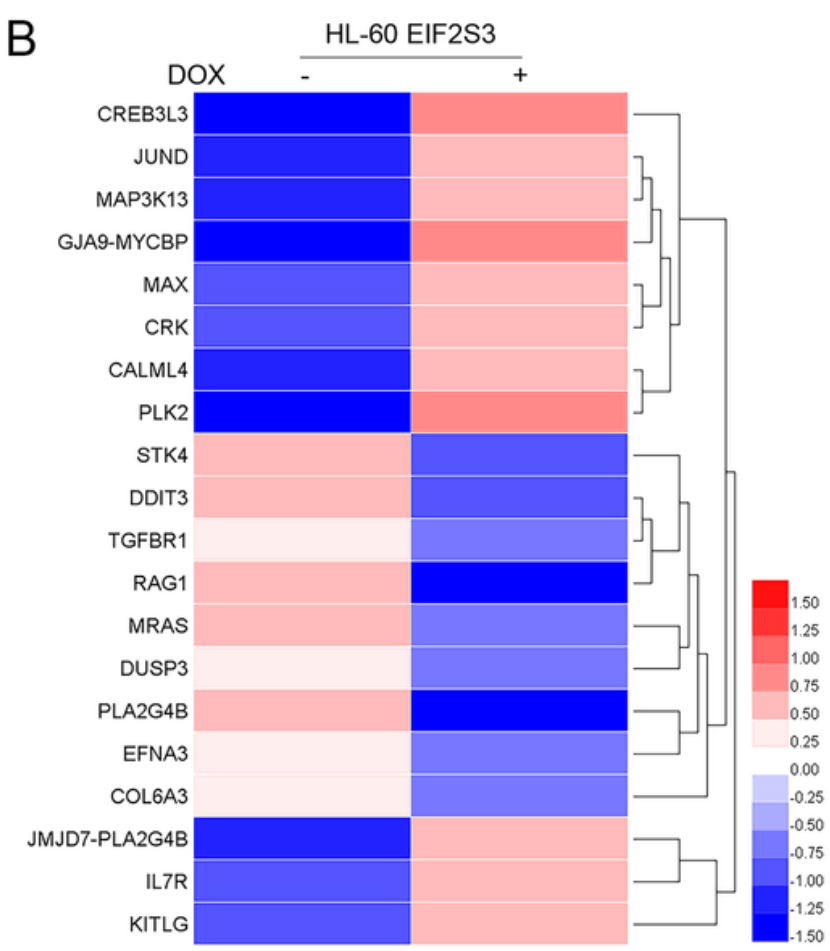

\section{Figure 5}

Analysis of five cell signaling pathways based on the results of KEGG analysis. (A) A total of five cell signaling pathways were chosen from Fig. 4 to further investigate the involved DEGs. (B) Heatmap of 20 DEGs; the closer the color to the ends of the color column, the greater the difference. KEGG, Kyoto Encyclopedia of Genes and Genomes; DEG, differentially expressed gene; DOX, doxycycline.

A

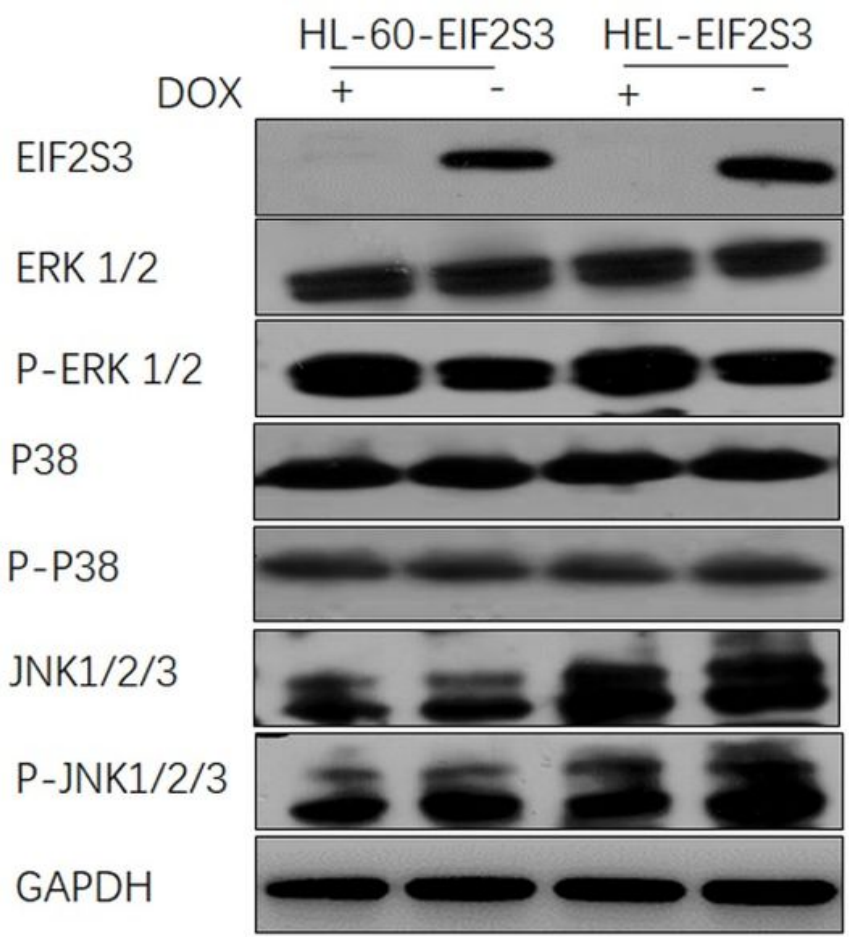

B
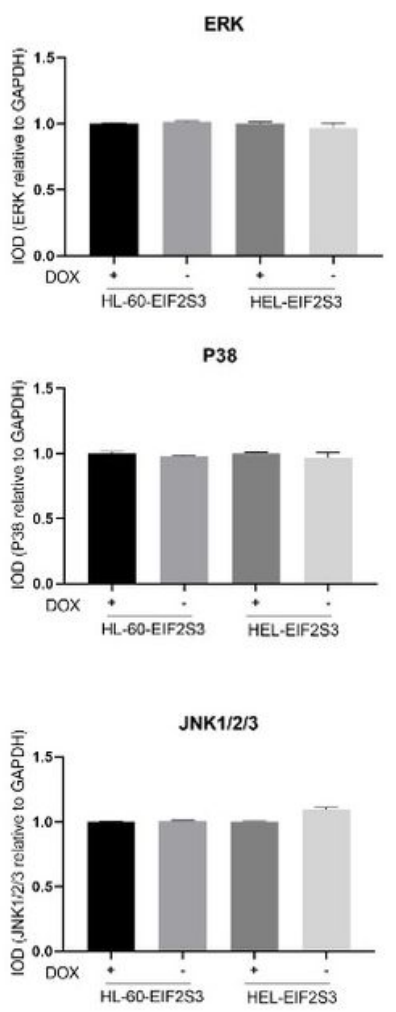
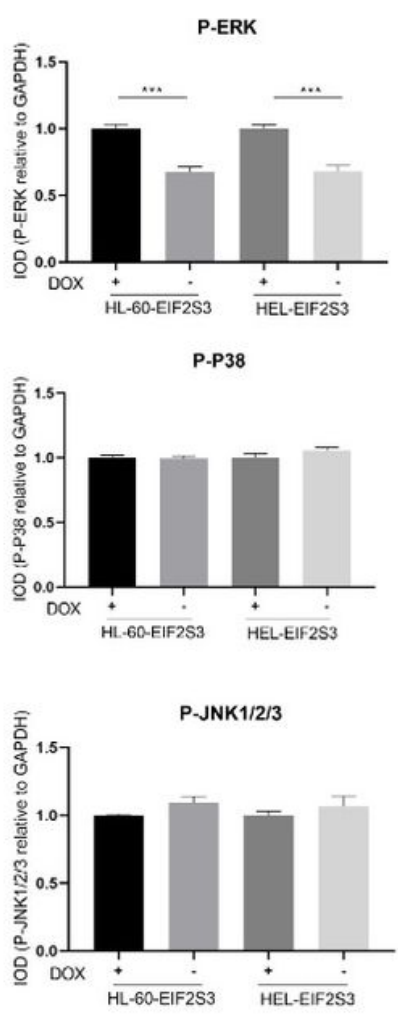


\section{Figure 6}

Relevant proteins of the MAPK signaling pathway and their phosphorylation levels in HEL-EIF2S3 and HL-60-EIF2S3 cells. (A) Western blot analysis of the relevant proteins and their phosphorylation levels in the MAPK signaling pathway. (B) Grayscale analysis of relative protein expression. Data are presented as the mean $\pm \mathrm{SEM}$. ${ }^{\star \star *} \mathrm{P}<0.001$. DOX, doxycycline.

\section{Supplementary Files}

This is a list of supplementary files associated with this preprint. Click to download.

- FigS1.tif

- Figs2.tif

- FigS3.tif

- FigS4.tif

- Figs5.tif

- FigS6.tif 